\title{
Autosomal sdY Pseudogenes Explain Discordances Between Phenotypic Sex and DNA Marker for Sex Identification in Atlantic Salmon
}

\author{
Fernando Ayllon ${ }^{1 *}$, Monica Favnebøe Solberg ${ }^{1}$, François Besnier ${ }^{1}$, Per Gunnar Fjelldal', \\ Tom Johnny Hansen ${ }^{1}$, Anna Wargelius ${ }^{1}$, Rolf Brudvik Edvardsen ${ }^{1}$ and \\ Kevin Alan Glover ${ }^{1,2}$
}

${ }^{1}$ Institute of Marine Research, Bergen, Norway, ${ }^{2}$ Department of Biological Sciences, University of Bergen, Bergen, Norway

\section{OPEN ACCESS}

Edited by:

Jacob A. Tennessen, Harvard University, United States

Reviewed by: Mark Kirkpatrick, University of Texas at Austin,

United States

Garrett McKinney, University of Washington,

United States

Kiyoshi Kikuchi,

The University of Tokyo, Japan

${ }^{*}$ Correspondence:

Fernando Ayllon

fernando.ayllon@hi.no

Specialty section:

This article was submitted to Evolutionary and Population Genetics,

a section of the journal Frontiers in Genetics

Received: 19 March 2020 Accepted: 17 September 2020 Published: 14 October 2020

Citation:

Ayllon F, Solberg MF, Besnier F, Fjelldal PG, Hansen TJ, Wargelius A, Edvardsen RB and Glover KA (2020) Autosomal sdY Pseudogenes Explain Discordances Between Phenotypic Sex and DNA Marker for Sex Identification in Atlantic Salmon.

Front. Genet. 11:544207. doi: 10.3389/fgene.2020.544207
Despite the key role that sex-determination plays in evolutionary processes, it is still poorly understood in many species. In salmonids, which are among the best studied fishes, the master sex-determining gene sexually dimorphic on the Y-chromosome (sdY) has been identified. However, sdY displays unexplained discordance to the phenotypic sex, with a variable frequency of phenotypic females being reported as genetic males. Multiple sex determining loci in Atlantic salmon have also been reported, possibly as a result of recent transposition events in this species. We hypothesized the existence of an autosomal copy of $s d Y$, causing apparent discordance between phenotypic and genetic sex, that is transmitted in accordance with autosomal inheritance. To test this, we developed a qPCR methodology to detect the total number of $s d Y$ copies present in the genome. Based on the observed phenotype/genotype frequencies and linkage analysis among 2,025 offspring from 64 pedigree-controlled families of accurately phenotyped Atlantic salmon, we identified both males and females carrying one or two autosomal copies of $s d Y$ in addition to the $Y$-specific copy present in males. Patterns across families were highly consistent with autosomal inheritance. These autosomal $s d Y$ copies appear to have lost the ability to function as a sex determining gene and were only occasionally assigned to the actual sex chromosome in any of the affected families.

Keywords: sex, evolution, autosome, pseudo-gene, SNP, Atlantic salmon, sdY, sex chromosome

\section{INTRODUCTION}

Most eukaryotic organisms reproduce sexually, yet the nature of the sexual system and the mechanism of sex determination often vary remarkably, even among closely related species (Ashman et al., 2014; Pennell et al., 2018). This is particularly true for teleosts where some species display genetic sex determination, some display environmental sex determination, while others a mixture of both (Heule et al., 2014). Furthermore, heterogametic systems for both male (XX females and XY males) and females (ZZ males and ZW females) are even found in closely related species of tilapias (Cnaani et al., 2008) or sticklebacks (Ross et al., 2009).

Atlantic salmon (Salmo salar) is an anadromous fish inhabiting temperate streams in the North Atlantic. It belongs to the family Salmonidae, which includes multiple species from 11 genera including salmon, trout, charr, freshwater whitefishes, ciscoes, and graylings. Globally, Atlantic salmon represents one of the most economically significant and iconic species, providing extensive 
angling recreation, large aquaculture production, and symbolizing healthy ecosystems in the rivers it inhabits. As a consequence, it is also one of the most exhaustively studied fish. The Atlantic salmon's ancestor underwent a whole-genome duplication event approximately 88-103 million years ago (Macqueen and Johnston, 2014), and is now in the process of rediploidization. As a result of this process, the Atlantic salmon genome consists of many paralogous regions (Lien et al., 2016) which in principle can diversify (Kjaerner-Semb et al., 2016) and acquire new functions as has been observed in other species displaying duplicated genomes (Qian and Zhang, 2014). Interestingly, the presence of transposable elements found in the genome is among the highest found in vertebrates (Lien et al., 2016).

The master sex determining (MSD) gene in salmonids is sexually dimorphic on the Y-chromsome ( $s d Y)$, and was first discovered in rainbow trout (Oncorhynchus mykiss) (Yano et al., 2012, 2013). The discovery of $s d Y$, and the subsequent development of molecular assays for rapid genetic sex determination has opened novel possibilities. For example, assays have been used to determine genetic sex in adults that were not phenotyped but subsequently used for sex-specific studies such as investigation into the genetic basis of age at maturity (Ayllon et al., 2015; Barson et al., 2015; Kusche et al., 2017; Ayllon et al., 2019). However, several studies have reported a discordance between phenotypic and $s d Y$ sex within the Salmonidae family (Eisbrenner et al., 2014; Cavileer et al., 2015; Larson et al., 2016; Podlesnykh et al., 2017). Discordance between DNA markers for sex and phenotypic sex is not uncommon in fishes, and it is typical in a species displaying a combination of genetic and environmental sex determination (Hattori et al., 2019). However, environmental sex determination has not been reported in the family Salmonidae, and several alternative theories for this discordance have been put forward including phenotyping errors (Yano et al., 2013; Eysturskarð et al., 2017), sex reversal (Nagler et al., 2001; Williamson and May, 2002; Metcalf and Gemmell, 2006), loss of gene function (Podlesnykh et al., 2017), or dose effects (Brown et al., 2020) among others (Guyomard et al., 2014; Larson et al., 2016; King and Stevens, 2020). Nevertheless, the mechanisms underpinning this discordance are still unclear. Adding to the complexity of the situation is the fact that $s d Y$ has been mapped to different regions of the genome in the various salmonid species, but also within the same species, suggesting that it transposes to a new location either at the time of speciation (Phillips, 2013) or more recently within species (Kijas et al., 2018). Specifically within Atlantic salmon, $s d Y$ has been mapped to chromosomes Ssa02, Ssa03, Ssa06, and possibly Ssa21 (Eisbrenner et al., 2014; Lubieniecki et al., 2015; Kijas et al., 2018; Gabian et al., 2019) evidencing its transposition ability (Lubieniecki et al., 2015).

In this study, we have identified why the presence of $s d Y$ does not always correlate with maleness in Atlantic salmon. We first asked the question whether the observed discordance could be linked to non-functional copies of $s d Y$ in the genome. We thereafter answered this by quantifying multiple $s d Y$ copies in the genome using qPCR on genomic DNA from 2,025 accurately phenotyped Atlantic salmon originating from 64 families of domesticated, F1-hybrid, and wild origin. We therefore demonstrate that the $s d Y$ gene has an infrequent non-functional copy in the genome, consistent with autosomal inheritance, which explains the observed discordance in Atlantic salmon females.

\section{MATERIALS AND METHODS}

\section{Experimental Crosses}

Over the past decade, we have conducted a number of pedigreecontrolled studies on a multiple-generation experimental population of domesticated and wild Atlantic salmon and their crosses at the aquaculture facility owned by the Institute of Marine Research located in Matre, western Norway (Solberg et al., 2013, 2014; Ayllon et al., 2015; Harvey et al., 2016, 2018; Glover et al., 2018, 2020; Perry et al., 2019; Besnier et al., 2020). The reader is directed to these publications for full details regarding the standard rearing conditions experienced in this fish farm. In the present study, we produced a total of 29 (F1-C2011) and 39 (F1-C2012) experimental families in the years 2011 and 2012, respectively. These families originated from the domesticated Mowi strain (13 families), the wild Figgjo population (14 families), reciprocal F1-hybrids between Mowi and Figgjo (24 families), the wild Vosso population (7 families), and the wild Arna population (6 families). Extensive details of these experimental crosses and the background of the source populations are available elsewhere (Solberg et al., 2014).

After fertilization in 2010 and 2011, eggs were incubated in single-family containers until the eyed stage when they were mixed into common-garden experiments to study a range of phenotypic traits (data not used here). These fish were first reared until smoltification in freshwater aged $1+$ when 2,000 (F1-C2011) and 2,400 (F1-2012) individuals were PIT tagged and DNA sampled, and thereafter transferred into sea-cages where they were reared until they matured after a further 13 years. Families represented by less than 10 individuals at maturity were discarded. Upon maturation, the phenotypic sex of 2,025 individuals from 64 families was accurately recorded by dissection, giving a total of 1,048 and 977 phenotypically validated males and females, respectively.

\section{Genetic Analysis-Microsatellites and SNPs}

Total DNA from all offspring and parents was purified using the Qiagen DNeasy Blood \& Tissue Kit (Qiagen, Hilden, Germany) according to the manufacturer's recommendations. Microsatellite DNA parentage testing was used to identify the pedigree of all individuals used in this study using the exclusion based method implemented in FAP (Taggart, 2007) using six microsatellites. Following the above-mentioned procedure, 97$99 \%$ of the offspring were unambiguously assigned to their family of origin. The laboratory conducting these analyses has extensive experience in DNA parentage testing (Solberg et al., 2013, 2014; Harvey et al., 2016, 2018; Glover et al., 2018), and the full details regarding the markers used and their amplification conditions are available in these previous studies. 
In addition to microsatellites, a set of 116 genome-wide distributed SNPs were genotyped in all offspring and parents for the purpose of linkage mapping (see below). This analysis was performed on a MassARRAY Analyzer 4 from Agena Bioscience $^{\mathrm{TM}}$ according to the manufacturer's instructions. The final dataset for mapping included 109 genome-wide distributed SNPs once those displaying poor coverage and clustering were removed. The list of SNPs are available elsewhere (Besnier et al., 2015, 2020) and their genomic location can be retrieved from the article describing the Atlantic salmon linkage map (Lien et al., 2011).

\section{PCR-Based sdY Tests}

The $s d Y$ presence/absence was validated by a PCR-based methodology aimed to detect the presence of the $s d Y$ gene (Yano et al., 2012; Eisbrenner et al., 2014). Individuals showing amplicons of exon 2 and 4 were designated as males. As a positive PCR control and for species determination we used the presence of the 5S rRNA gene (Pendas et al., 1995). PCR amplifications were performed using reaction mixtures containing approximately $50 \mathrm{ng}$ of extracted Atlantic salmon DNA, 10 nM Tris-HCl pH 8.8, 1.5 mM MgCl2, 50 mM KCl, 0.1\% Triton X-100, $0.35 \mu \mathrm{M}$ of each primers, 0.5 Units of DNA Taq Polymerase (Promega, Madison, WI, United States) and $250 \mu \mathrm{M}$ of each dNTP in a final volume of $20 \mu \mathrm{L}$. PCR products were visualized in 3\% agarose gels.

A quantitative PCR (qPCR) based methodology was developed to quantify the number of $s d Y$-liked copies present. gapdh, $s d Y$ exon 2, and $s d Y$ exon4 were multiplexed using 5'labeled probes (Supplementary Table 1). The gapdh locus was used as an internal positive control (IPC) and reference gene to estimate fold change (FC) values (Livak and Schmittgen, 2001). Amplification reactions were run on a QuantStudio5 384 real time detection system (Thermo Fisher Scientific, United States). Reactions consisted of a Pre-Read stage $\left(60^{\circ} \mathrm{C}\right.$ for $30 \mathrm{~s}$ ), a Hold Stage $\left(95^{\circ} \mathrm{C}\right.$ for $\left.10 \mathrm{~min}\right)$, a PCR stage (40 cycles of $95^{\circ} \mathrm{C}$ for $15 \mathrm{~s}$ and $60^{\circ} \mathrm{C}$ for $1 \mathrm{~min}$ ) and PostRead stage $\left(60^{\circ} \mathrm{C}\right.$ for $\left.30 \mathrm{~s}\right)$. Each $5 \mu \mathrm{l}$ reaction contained the following final concentrations: $1 \times$ Taqman Universal MasterMix, $1 \mu \mathrm{M}$ gapdh forward and reverse primers, 0.2 $\mu \mathrm{M}$ gapdh TaqMan probe, $1.4 \mu \mathrm{M} s d Y \_$Exon2 forward and reverse primers, $0.32 \mu \mathrm{M} s d Y$ _Exon2 TaqMan probe, $2.1 \mu \mathrm{M}$ $s d Y \_$Exon4 forward and reverse primers, $0.48 \mu \mathrm{M} s d Y \_$Exon4 TaqMan probe and $2 \mathrm{ng} / \mu \mathrm{l}$ of gDNA template. Whenever possible, no template controls (NTC) and reference males and females were included.

In order to validate the qPCR methodology, we used XY males and YY super-males. XY males will carry a single copy of the Y-specific sex determining gene $s d Y$. On the other hand, super males will carry two $s d Y$ gene copies, one per Y chromosome. YY males are the product of either self-fertilization or double haploid males. Full details on YY super-males production can be found in Fjelldal et al. (under review). Briefly, eggs and milt from a hermaphrodite salmon were surgically removed to prevent undesired self-fertilization. Eggs were then selffertilized either with normal or UV-irradiated milt. Following the fertilization with UV-irradiated milt, pressure mediated diploidization was carried out to produce the double haploid males used in this study.

\section{Linkage Mapping}

Linkage mapping was performed on all 64 families, including the eight families showing discrepancy between genetic and phenotypic sex. For each family, the coefficient of Identity By Descent (IBD) among offspring alleles was estimated from both pedigree and genotype information as in Pong-Wong et al. (2001). First, the genomic location of the sex determining locus was considered. The link between the binary phenotype (male/female) and the two paternally (maternally) inherited alleles was investigated by fitting a Chi-squared test in each family separately, at each SNP locus. Second, the genomic location of the sex discrepancy for the eight affected families was investigated following the same Chi-squared approach, also at the family level. Here, the two phenotypes were no longer male and female but discrepant/non-discrepant individuals, where the non-discrepant group consisted of all the regular males and females, and the discrepant group consisted of phenotypic females that amplified one or more copy of $s d Y$, as well as phenotypic males that amplified more than one $s d Y$ copy.

\section{Statistical Analysis}

Chi square tests with computed $p$-values by Monte Carlo simulations $\left(10^{6}\right.$ replicates) were used to test for deviations of the observed values from the expected $s d Y$ genotypes frequencies. All statistical analyses were conducted in R V.3.6.2. (R Development Core Team, 2019).

\section{Ethical Considerations and Research Permits}

The experimental protocols (permit numbers 4268, 5296) were approved by the Norwegian Animal Research Authority (NARA). Use of experimental animals were performed in strict accordance with the Norwegian Animal Welfare Act. This included anesthesia or euthanasia of fish using metacain (Finquel ${ }^{\circledR}$ Vet, ScanVacc, Årnes, Norway), during all described procedures. In addition, all personnel involved in this experiment had undergone training approved by the Norwegian Food Safety Authority, which is mandatory for all personnel running experiments involving animals included in the Animal Welfare Act.

\section{RESULTS}

We found PCR-based discordance between the validated phenotypic sex and $s d Y$ genotype in 66 individuals out of the 2,025 fish from the 64 families tested (Figure 1). All of the reported cases were phenotypic females displaying a positive signal for $s d Y$. Discordance between the $s d Y$ presence/absence and phenotypic sex was only observed in females from eight of the 64 families, ranging from 36 to $82 \%$ discordance among females per family. Of the 88 parents used as broodstock, three phenotypic females were $s d Y$ positive. These females were the mothers of families F26, F27 and K22, all of which had female 


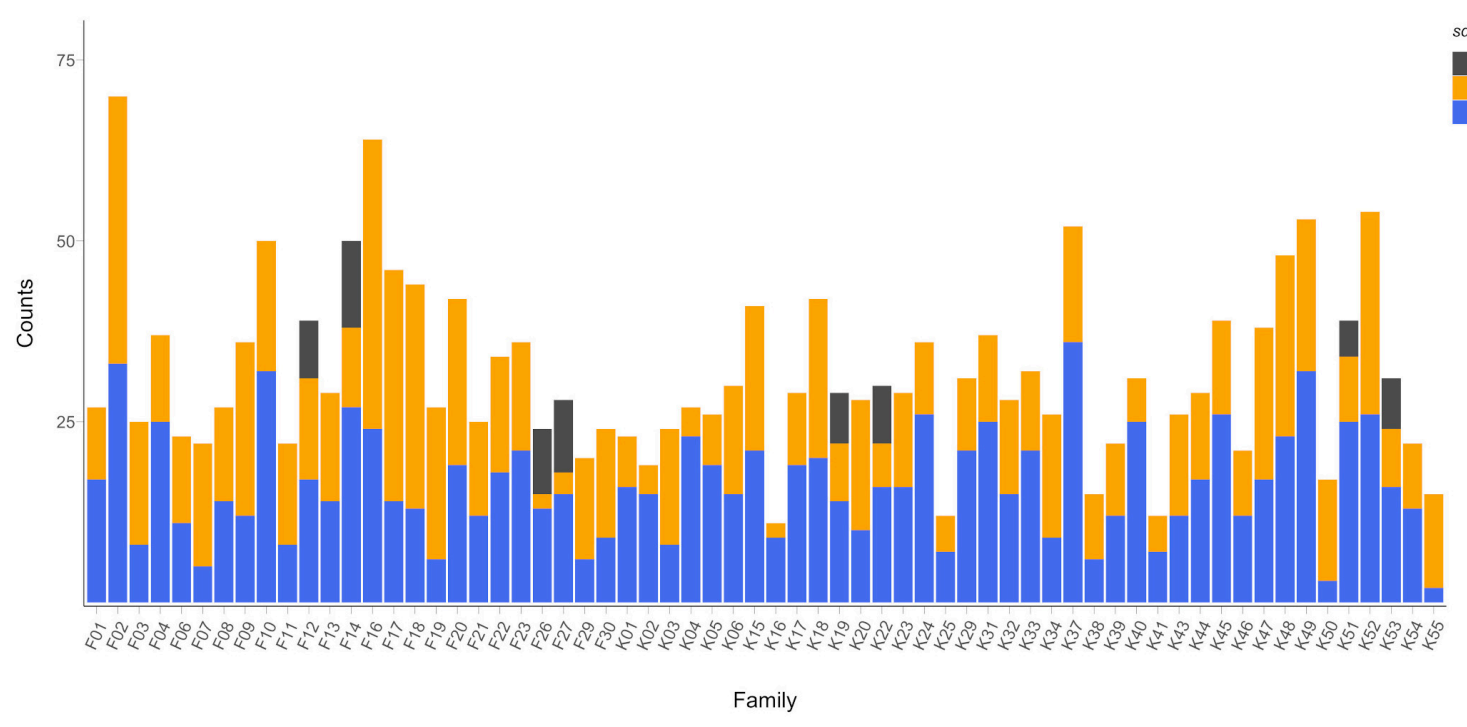

FIGURE 1 | Phenotypic sex frequencies distribution across the 64 studied families showing PCR-based sdY genetic sex concordances. Concordant males and females are displayed in blue and orange, respectively. Discordant females (sdY positive phenotypic females) are shown in dark gray.

offspring displaying discordance between phenotypic and genetic sex (Figure 1). The five other families containing discordant offspring did not have discordant mothers.

Based on the above result, we hypothesized that some Atlantic salmon may display a second autosomic copy of $s d Y$ in the genome. To examine this possibility, we used FC values from the qPCR assay in order to investigate the number of copies of the $s d Y$ present in each individual (both the sex determining gene and the potential autosomic pseudocopy). First, we genotyped known XY and YY males in order to validate the potential to identify two copies of this gene using the assay (Figure 2A). This test demonstrated that XY males clustered around $1 \mathrm{FC}$ values for both amplicons (exons 2 and 4). In addition, YY males, i.e., males containing two $s d Y$ copies, all clustered around a FC value of 2 ( $>1.5$ FC threshold for both exons). All three discordant dams described above carried a single $s d Y$ copy while there were four sires that were identified as carrying two copies of $s d Y$ (Figure 2B). Significantly, these four males sired the five families displaying discordant offspring that did not have a discordant dam, and in addition, sired two families with a discordant dam (Supplementary Table 2). Thus, at this stage, it was demonstrated that all eight families displaying discordant offspring had one or two discordant parents. Furthermore, none of the 56 families without discordant offspring had discordant parents. Among the 2,025 offspring, 62 and 4 of the phenotypic females displayed one and two copies of $s d Y$, respectively, and, 66 and eight of the phenotypic males displayed two and three copies of $s d Y$, respectively (Figure 2C). All of these individuals were reported from the eight families with offspring displaying discordance between phenotypic and genetic sex. When these data were considered on a family by family basis, the observed frequencies of the offspring carrying a variable number of copies of the $s d Y$ gene were highly consistent with autosomal inheritance (Figures 3, 4).
Based upon our hypothesis developed above, and the observed parental $s d Y$ genotypes in six of the families, we expected to see a $50 / 50$ frequency in the female offspring displaying $0 \times$ vs. $1 \times s d Y$ copies, and the same frequency in male offspring displaying $1 \times$ vs. $2 \times s d Y$ copies. The observed frequencies (Figure 3) did not deviate from the expected frequencies (Chi Square $p$-values ranging from 0.24 to 0.98 ). In the remaining two families, and based upon the parental genotypes, we expected to see a $25 / 50 / 25$ distribution in the frequencies $0 \times, 1 \times, 2 \times$, and $1 \times, 2 \times$, or $3 \times$ copies of $s d Y$ for female and male offspring, respectively. Although the observed offspring frequencies did not match exactly with these expected frequencies (Figure 4), most likely due to very low $\mathrm{N}$ offspring within these families, they did not significantly deviate from the expected frequencies ( $p$-values 0.29 and 0.34 from families 26 and 27, respectively).

Within the 64 families, validated phenotypic sex was mapped to chromosomes Ssa02, Ssa03 and Ssa06 (Supplementary Table 2). Thereafter, the offspring from the eight affected families, with their $s d Y$ genotype (i.e., females displaying $0 \times$ vs. $1 \times$ or $2 \times s d Y$ copies, and males displaying $1 \times$ vs. $2 \times$ or $3 \times s d Y$ copies), was mapped to chromosomes Ssa03, Ssa05, Ssa06, Ssa13 and Ssa28. Statistical support was however variable for some of the mapping data, in part possibly due to low $\mathrm{N}$ observations (Supplementary Table 2).

\section{DISCUSSION}

The discovery of the master sex-determining $s d Y$ gene and its function in salmonids represents a significant advance in knowledge (Yano et al., 2012, 2013; Bertho et al., 2018), opening up new avenues of research. However, phenotype$s d Y$ discordances have been reported in many of the salmonid species, which has left open questions regarding $s d Y$ in salmonids 
A

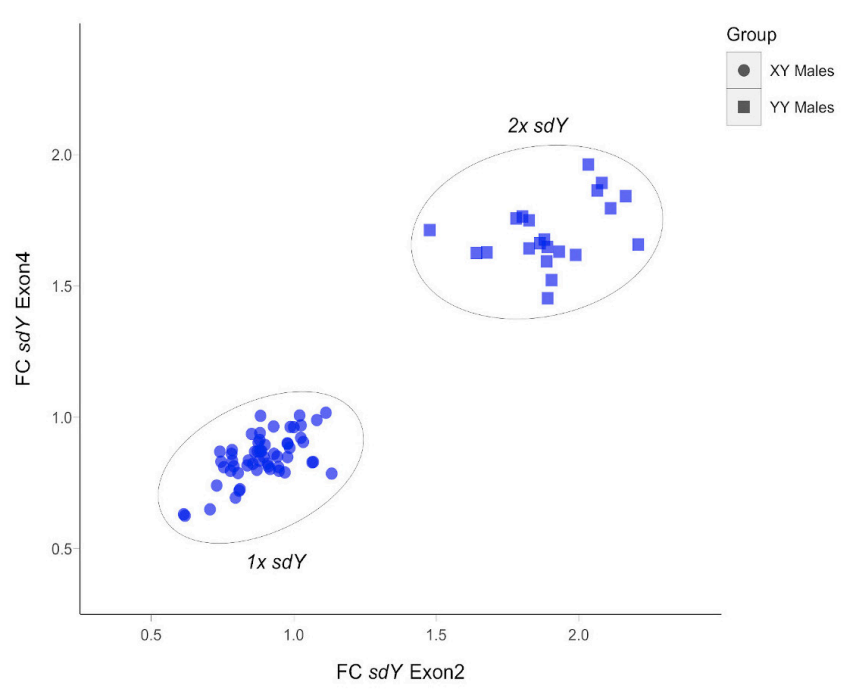

B

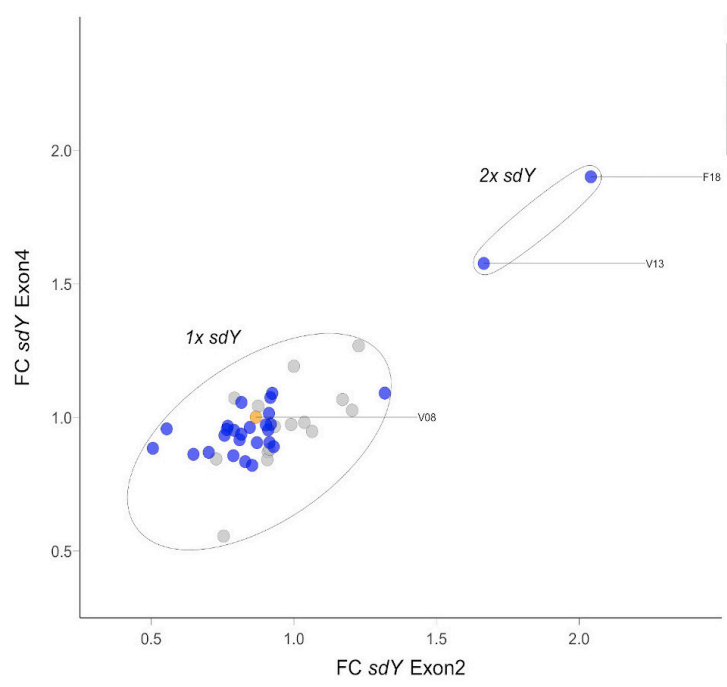

C

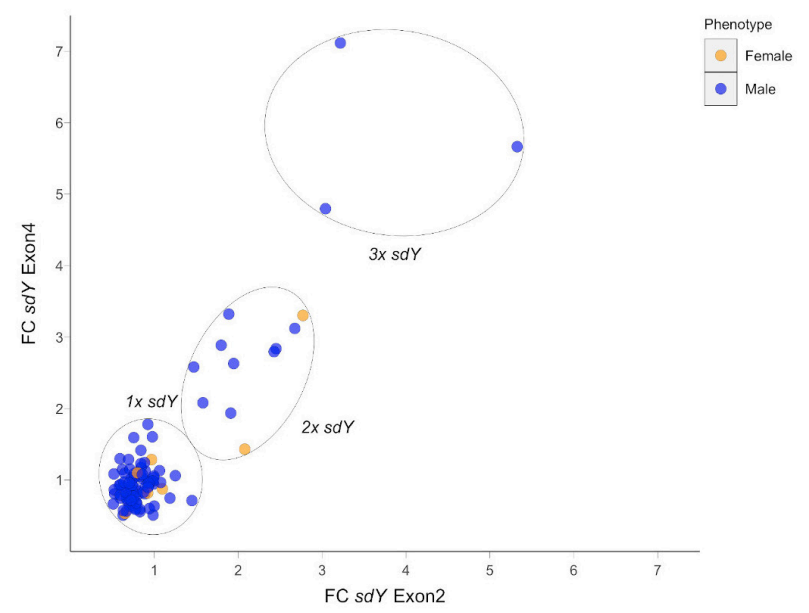

FIGURE 2 | Real time PCR based fold change (FC) values for sdY exon2 and exon4 amplicons. (A) Validation RT PCR using XY males and YY super-males; round and square dots, respectively. (B) Parental individuals for the F1-K2012 families showing $1-2 \times s d Y$ sires individuals, in blue, and the $1 \times s d Y$ discordant dam in orange. Reference $1 \times s d Y$ males are displayed in gray. (C) Example plate showing 1-3x sdY males and $1-2 \times s d Y$ females in blue and orange, respectively, among the offspring. Reference males not displayed. 


\section{A Phenotype}

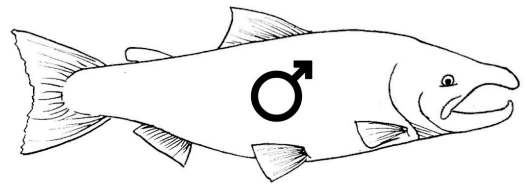

$\mathbf{X}$

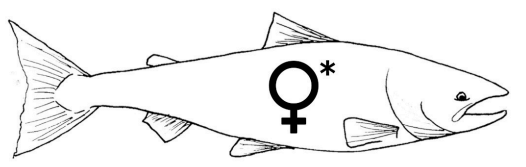

B Inheritance Model
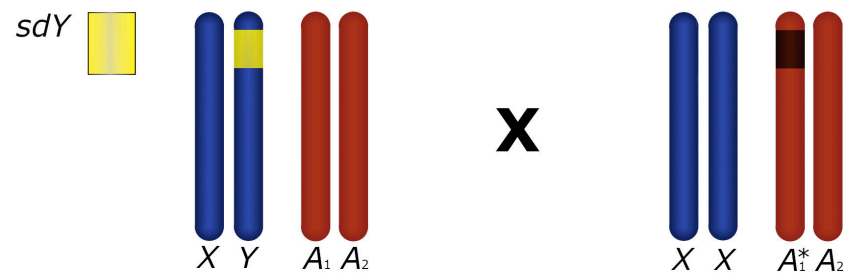

Pseudo sdY

c Expected proportions
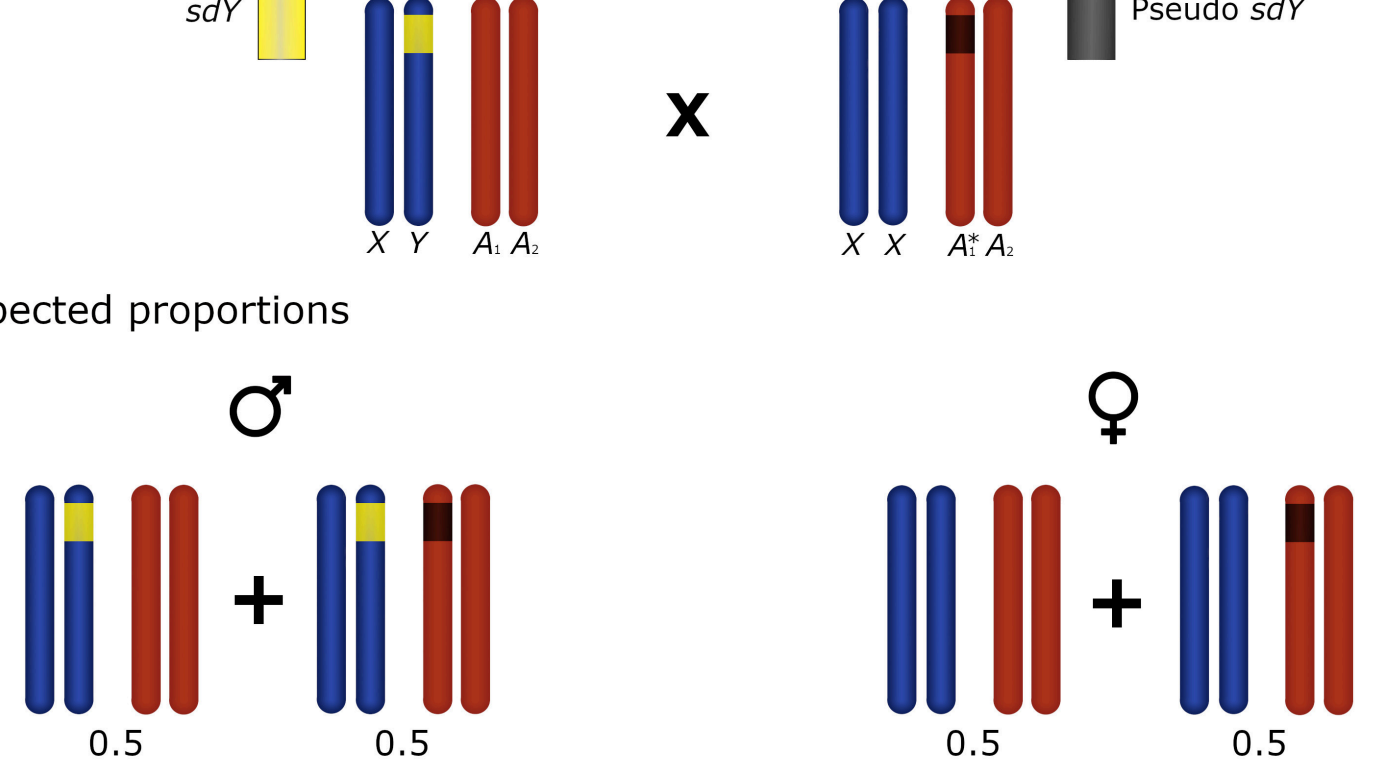

D Observed proportions

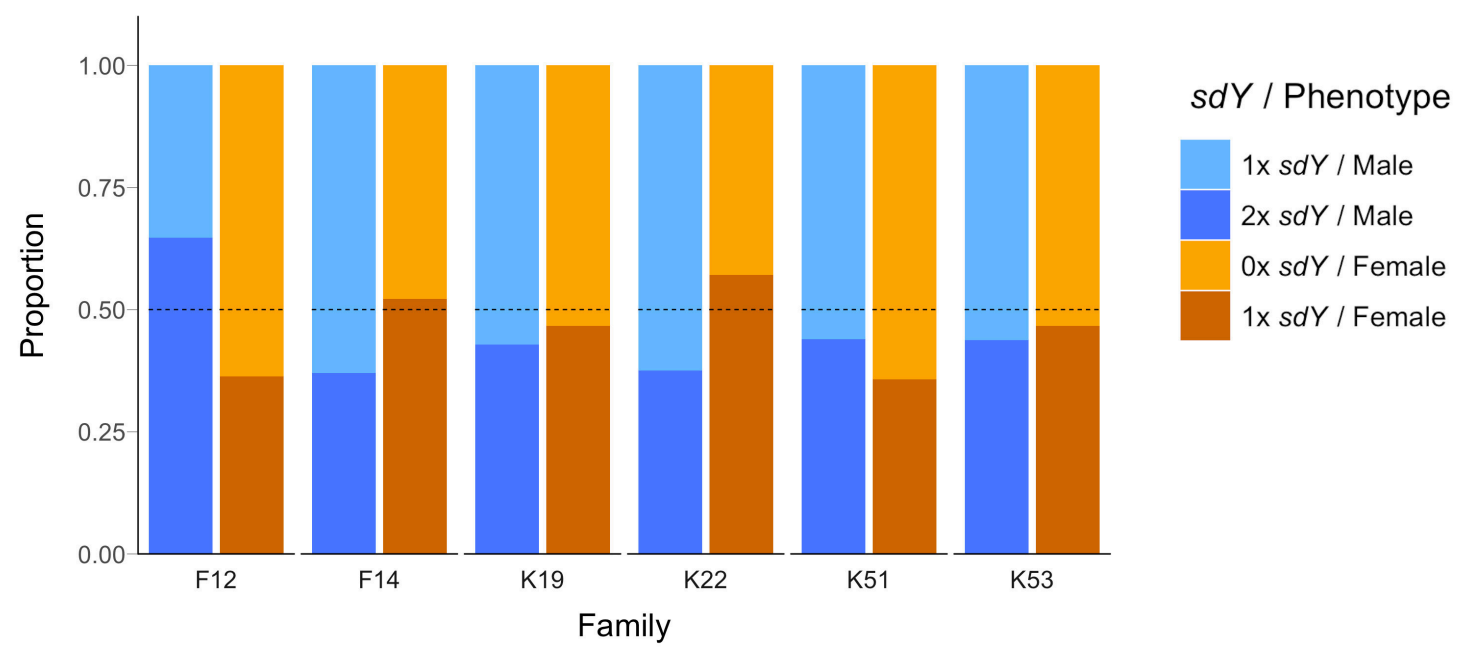

FIGURE 3 | Conceptual diagram showing the inheritance model and the observed frequencies for the offspring of crosses with one parental individual carrying an $s d Y$ pseudocopy. (A) Cross between a $1 \times s d Y$ phenotypic male and a $1 \times s d Y$ discordant female denoted with *. (B) conceptual diagram showing the inheritance model at a chromosome level. Sex chromosomes and autosomes are represented in dark blue and dark orange, respectively. Normal $1 \times s d Y$ male (left) carrying a copy of the sex determining $s d Y$ gene (yellow) in the $Y$ chromosome. Discrepant $1 \times s d Y$ phenotypic female (right) carrying an sdY autosomic pseudocopy (dark gray) in heterozygosis. (C) Males and females expected proportions for the different $s d Y$ genotypes (1-2x). (D) Offspring observed proportions for the affected families of $1 \times$ and $2 x s d Y$ males and $0 x$ and $1 \times s d Y$ females: light blue, blue, light orange and orange, respectively. Dotted lines represent the sdY genotype expected frequencies. 


\section{A Phenotype}

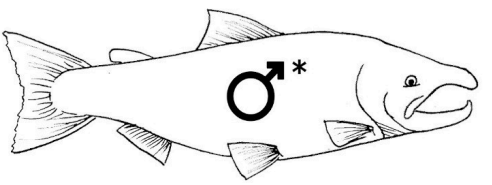

$\mathbf{x}$

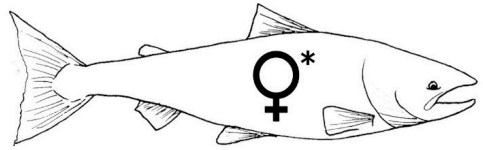

B

Inheritance Model
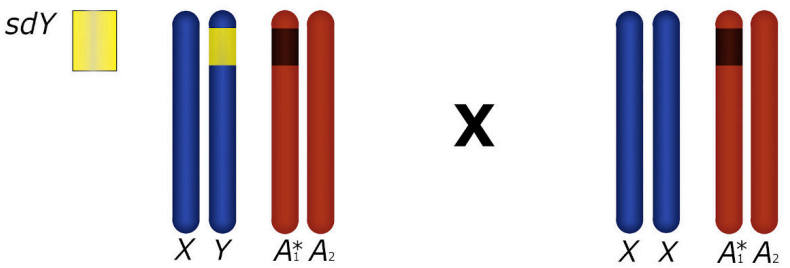

Pseudo sdY

c Expected proportions

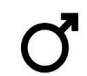

우

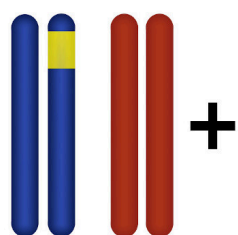

0.25

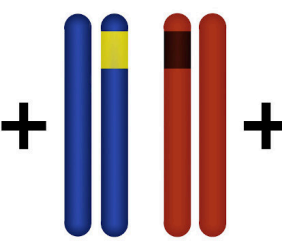

0.5

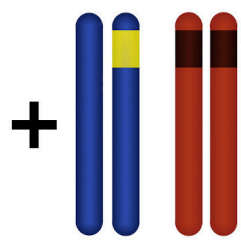

0.25

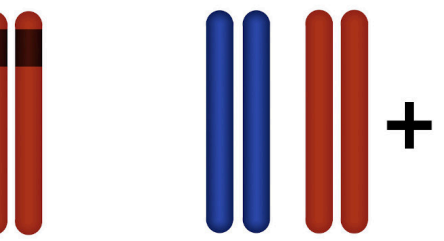

0.25

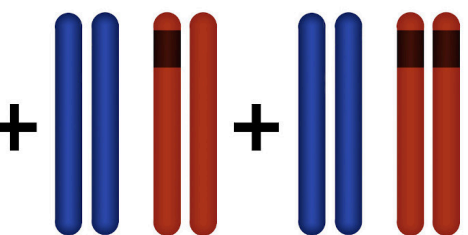

0.5

0.25

D Observed proportions

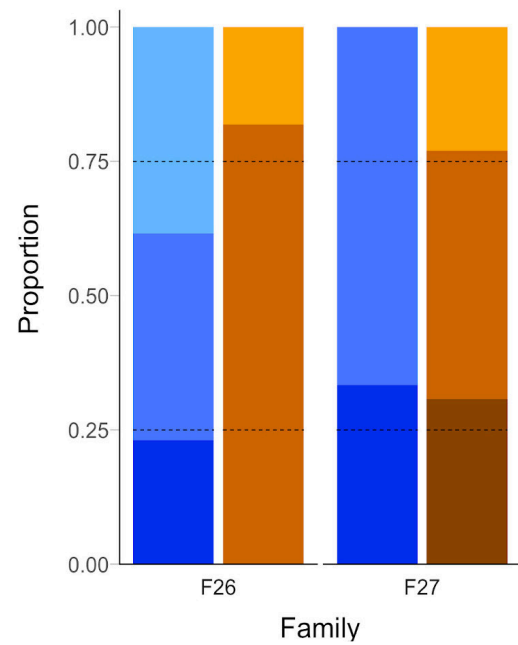

sdY / Phenotype

1X $s d Y /$ Male

$2 \mathrm{x} s d \mathrm{Y} / \mathrm{Male}$

$3 \mathrm{x}$ sdy / Male

$0 \mathrm{x} s d \mathrm{Y} /$ Female

$1 \mathrm{x} s d \mathrm{Y} /$ Female

$2 \mathrm{x} s d Y /$ Female

FIGURE 4 | Conceptual diagram showing the inheritance model and the observed frequencies for the offspring of crosses with both parental individuals carrying an sdY pseudocopy. (A) Cross between a $2 \times s d Y$ phenotypic male and a $1 \times s d Y$ discordant female: Both affected parental individuals are denoted with an asterisk. (B) Conceptual diagram showing the inheritance model at a chromosome level. Sex chromosomes and autosomes are represented in dark blue and dark orange, respectively. (B) $2 \times s d Y$ male (left) carrying a copy of the sex determining sdY gene (yellow) in the $Y$ chromosome and the extra autosomic copy (dark gray) in heterozygosis. Discrepant $1 \times s d Y$ phenotypic female (right) carrying an sdY autosomic pseudocopy (dark gray) in heterozygosis. (C) Males and females expected proportions for the different $s d Y$ genotypes (1-3x). (D) Offspring observed proportions for the affected families of 1-3x sdY males and $0-2 \times s d Y$ females: light blue, blue, dark blue, light orange, orange, and dark orange, respectively. Dotted lines represent the sdY genotype expected frequencies. 
(Yano et al., 2012; Cavileer et al., 2015; Larson et al., 2016). Here, we have presented extensive and compelling data that strongly suggest that these discordances in Atlantic salmon are caused by low-frequency $s d Y$ copies in the genome that are not involved in sex determination.

Within the genetic material studied here, we observed $6.75 \%$ discordant females, and a total of $4 \%$ of the individuals with a second or third copy of the pseudo $s d Y$ gene. These fish originated from three dams and five sires among the 88 parents. The number of discordant females observed here is higher than the $1 \%$ frequency observed in a domesticated Tasmanian Atlantic salmon strain (Eisbrenner et al., 2014; Kijas et al., 2018) but similar to the $7 \%$ recently reported for the same Tasmanian strain (Brown et al., 2020). Given the inheritance model presented here, it is likely that this difference is merely the result of the number of affected parents and the cross design, although strain specific differences in the frequency of the pseudocopy of $s d Y$ cannot be ruled out. The 7 and 12\% discordances between phenotype and $s d Y$ marker for sex reported in sockeye salmon (Larson et al., 2016) and the chinook salmon (Cavileer et al., 2015), respectively, raise the question of whether the same phenomenon described in Atlantic salmon here is also the cause of observed discordance in the other salmonid species. Members of the Coregoninae subfamily lack $s d Y$ male specificity and the existence of sex specific inactive copies in females has been invoked to explain this phenomenon (Yano et al., 2012). Together with the mobile nature of the $s d Y$ gene, the loss of function may also explain the existence of inactive $s d Y$ copies in other salmonid species (Podlesnykh et al., 2017).

Since the $s d Y$ gene was discovered (Yano et al., 2013), different mechanisms have been invoked to explain the existence of discordant phenotypes such as phenotyping or sampling errors, environment mediated sex reversal, female-specific gene inactivation, sequence variability, the existence of minor sex determining (SD) genes and recombination (Yano et al., 2013; Cavileer et al., 2015; Larson et al., 2016; Eysturskarð et al., 2017; King and Stevens, 2020). Recently, a dosage-dependent mechanism has been suggested to explain these discrepancies in Atlantic salmon (Brown et al., 2020), suggesting that $s d Y$ is present in a single copy in the male genome and might be also present as partial copies in the female genome. However, our results strongly point to the existence of non-functional autosomal copies as previously suggested for two coregoninae species (Yano et al., 2013) and sockeye salmon (Larson et al., 2016). Here we report the existence of phenotypic females with up to two full $s d Y$ autosomal copies which appear to have lost their ability to function as a proper SD gene causing the apparent discordance.

Within Atlantic salmon, $s d Y$ has been mapped to chromosomes Ssa02, Ssa03 and Ssa06 in domesticated and wild strain of salmon from North America and Norway (Eisbrenner et al., 2014; Kijas et al., 2018; Besnier et al., 2020). It has also been mapped to chromosomes Ssa02 and Ssa21 in six wild Spanish populations (Gabian et al., 2019). The above observations are consistent with the findings of the present study where the SD $s d Y$ was mapped to Ssa02, Ssa03, and Ssa06 in the 64 families. In all of these studies, chromosome Ssa02 was identified as the most common location for $s d Y$ and is likely to be the ancestral variant (Kijas et al., 2018). Surprisingly, low divergence between the Ssa03 and Ssa06 loci has been reported (Kijas et al., 2018), suggesting a recent origin even though these variants are present in both the North American and European lineages. Here, we mapped $s d Y$ autosomal copies to chromosomes Ssa03, Ssa05, Ssa06, Ssa13, and Ssa28. Interestingly however, within the eight families displaying $s d Y$ autosomal copies, SD $s d Y$, and autosomal $s d Y$ were only mapped on the same chromosome in family F12. Therefore, these two genes typically did not co-locate on chromosomes in the analyzed families. Ultimately, this might explain why only a limited number of chromosomes are recursively recruited as sex chromosomes in the species (Eisbrenner et al., 2014; Gabian et al., 2019), both recently and in different lineages (Kijas et al., 2018). The primers used here have been proven to be robust in detecting both $s d Y$ and its autosomal copy at the expected frequencies, so it is reasonable to infer a high degree of primer binding sequence conservation. Hence, it is also fair to reason that these pseudocopies may very well be the product of recent transpositions (Lubieniecki et al., 2015) and may constitute failed attempts to recruit novel sex determining loci in the species. Sex chromosome recruitment in Atlantic salmon may be the product of the transposable nature of the $s d Y$ gene (Faber-Hammond et al., 2015) and gene landscape (Bertho et al., 2018), which might explain the loss of function of the autosomal copies reported here. A functional $s d Y$ copy is considered necessary for maleness in salmonids but Atlantic salmon $s d Y$ negative males has been sporadically reported (Perry et al., 2019; Brown et al., 2020). However, they seem to be the product of PCR artifacts (King and Stevens, 2020).

Results of the present study, including the $s d Y$ copy number assay developed herein, have implications for commercial salmonid breeding programs. Breeders are increasingly using the $s d Y$ gene to determine phenotypic sex and to assist broodstock selection in the early production phase. Discordance between phenotypic and $s d Y$ based genetic tests has been reported for example in the commercial Mowi strain representing a logistic and financial challenge (Matt Baranski, Mowi, personal communication). Being able to identify both males and females carrying pseudocopies creates the opportunity to remove this pseudocopy from the breeding line in one generation: males carrying two or three copies and females carrying two copies can be removed early, and single copy carrying females weeded out when the phenotype is clear. Additionally, gaining knowledge about the proper genomic environment needed for a successful sex chromosome recruitment might constitute a huge leap in the race of understanding the precise mechanisms behind sex determination and ultimately in gaining control of the process from an aquaculture perspective.

\section{DATA AVAILABILITY STATEMENT}

The data is available at Open Science Framework with the next Identifier: DOI: 10.17605/OSF.IO/3XHA9 (https://osf.io/ $3 x h a 9 /)$. 


\section{ETHICS STATEMENT}

The animal study was reviewed and approved by the Norwegian Animal Research Authority (NARA) Permit numbers 4268 and 5296.

\section{AUTHOR CONTRIBUTIONS}

KG, MS, and FA conceived and designed the experiments. FA, MS, and PF performed the experiments. FA, MS, FB, and KG analyzed the data and contributed to the interpretation of the results. KG, $\mathrm{AW}, \mathrm{RE}, \mathrm{PF}$, and $\mathrm{TH}$ contributed to the reagents, materials, and analysis tools. FA and KG wrote the manuscript. All authors provided critical feedback and helped shape the research, analysis, and manuscript.

\section{FUNDING}

This work was funded by the Norwegian Ministry of Trade and Fisheries, and the Norwegian Research Council in the grants INTERACT (200510), MATGEN (254783), and POSTSMOLTMAT (254870). Neither funding body played any

\section{REFERENCES}

Ashman, T.-L., Bachtrog, D., Blackmon, H., Goldberg, E. E., Hahn, M. W., Kirkpatrick, M., et al. (2014). Tree of sex: a database of sexual systems. Sci. Data 1:140015. doi: 10.1038/sdata.2014.15

Ayllon, F., Kjaerner-Semb, E., Furmanek, T., Wennevik, V., Solberg, M. F., Dahle, G., et al. (2015). The vgll3 locus controls age at maturity in wild and domesticated Atlantic salmon (Salmo salar L.) males. PLoS Genet. 11:e1005628. doi: 10.1371/journal.pgen.1005628

Ayllon, F., Solberg, M. F., Besnier, F., Fjelldal, P. G., Hansen, T. J., Wargelius A., et al. (2020). Sex determining gene transposition as an evolutionary platform for chromosome turnover. bioRxiv doi: 10.1101/2020.03.14.991026

Ayllon, F., Solberg, M. F., Glover, K. A., Mohammadi, F., Kjaerner-Semb, E., Fjelldal, P. G., et al. (2019). The influence of vgll3 genotypes on sea age at maturity is altered in farmed mowi strain Atlantic salmon. BMC Genet. 20:44. doi: 10.1186/s12863-019-0745-9

Barson, N. J., Aykanat, T., Hindar, K., Baranski, M., Bolstad, G. H., Fiske, P., et al. (2015). Sex-dependent dominance at a single locus maintains variation in age at maturity in salmon. Nature 528, 405-408. doi: 10.1038/nature 16062

Bertho, S., Herpin, A., Branthonne, A., Jouanno, E., Yano, A., Nicol, B., et al. (2018). The unusual rainbow trout sex determination gene hijacked the canonical vertebrate gonadal differentiation pathway. Proc. Natl. Acad. Sci. U.S.A. 115, 12781-12786. doi: 10.1073/pnas.1803826115

Besnier, F., Glover, K. A., Lien, S., Kent, M., Hansen, M. M., Shen, X., et al. (2015). Identification of quantitative genetic components of fitness variation in farmed, hybrid and native salmon in the wild. Heredity 115, 47-55. doi: 10.1038/hdy. 2015.15

Besnier, F., Solberg, M. F., Harvey, A. C., Carvalho, G. R., Bekkevold, D., Taylor, M. I., et al. (2020). Epistatic regulation of growth in Atlantic salmon revealed: a QTL study performed on the domesticated-wild interface. BMC Genet. 21:13. doi: 10.1186/s12863-020-0816-y

Brown, M. S., Evans, B. S., and Afonso, L. O. B. (2020). Discordance for genotypic sex in phenotypic female Atlantic salmon (Salmo salar) is related to a reduced $s d Y$ copy number. Sci. Rep. 10:9651. doi: 10.1038/s41598-020-66406-x

Cavileer, T.D., Hunter, S.S., Olsen, J., Wenburg, J., and Nagler, J.J. (2015). A sex-determining gene $(s d Y)$ assay shows discordance between phenotypic and role in the design of the study, interpretation of data, nor conclusions drawn.

\section{ACKNOWLEDGMENTS}

We greatly acknowledge Lise Dyrhovden, Ivar Helge Matre, Kåre Storsaeter, and Jan Olav Fosse at the Matre research station (IMR) for rearing of fish included in this study, Anne Grete E. Sørvik and Zhiwei Zhang for microsatellite and SNP genotyping, and MOWI for unconditionally providing the domesticated gametes and for discussions regarding the implications of these results. We would also like to thank the river owners for access to wild broodstock. Emily K. Glover is gratefully acknowledged for drawing the salmon used in Figures 3, 4. This manuscript has been released as a pre-print at BioRxiv (Ayllon et al., 2020).

\section{SUPPLEMENTARY MATERIAL}

The Supplementary Material for this article can be found online at: https://www.frontiersin.org/articles/10.3389/fgene. 2020.544207/full\#supplementary-material

genotypic sex in wild populations of chinook salmon. Trans. Am. Fish. Soc. 144, 423-430. doi: 10.1080/00028487.2014.993479

Cnaani, A., Lee, B. Y., Zilberman, N., Ozouf-Costaz, C., Hulata, G., Ron, M., et al. (2008). Genetics of sex determination in tilapiine species. Sex. Dev. 2, 43-54. doi: 10.1159/000117718

Eisbrenner, W. S., Botwright, N., Cook, M., Davidson, E. A., Dominik, S., Elliott, N. G., et al. (2014). Evidence for multiple sex-determining loci in Tasmanian Atlantic salmon (Salmo salar). Heredity 113, 86-92. doi: 10.1038/hdy.2013.55

Eysturskarð, J., Dam, M., í Kongsstovu, S. K., Jacobsen, Á, and Petersen, P. E. (2017). Rapid sex identification of Atlantic salmon (Salmo salar L.) by real-time PCR. Aquac. Res. 48, 2618-2620. doi: 10.1111/are.13003

Faber-Hammond, J. J., Phillips, R. B., and Brown, K. H. (2015). Comparative analysis of the shared sex-determination region (SDR) among salmonid fishes. Genome Biol. Evol. 7, 1972-1987. doi: 10.1093/gbe/evv123

Gabian, M., Moran, P., Fernandez, A. I., Villanueva, B., Chtioui, A., Kent, M. P., et al. (2019). Identification of genomic regions regulating sex determination in Atlantic salmon using high density SNP data. BMC Genomics 20:764. doi: 10.1186/s12864-019-6104-4

Glover, K. A., Harvey, A. C., Hansen, T. J., Fjelldal, P. G., Besnier, F. N., Bos, J. B., et al. (2020). Chromosome aberrations in pressure-induced triploid Atlantic salmon. BMC Genet. 21:59. doi: 10.1186/s12863-020-00864-0

Glover, K. A., Solberg, M. F., Besnier, F., and Skaala, O. (2018). Cryptic introgression: evidence that selection and plasticity mask the full phenotypic potential of domesticated Atlantic salmon in the wild. Sci. Rep. 8:13966. doi: 10.1038/s41598-018-32467-2

Guyomard, R., Guiguen, Y., Bernard, M., Charlet, A., Dechamp, N., Hervet, C., et al. (2014). "RAD-seq mapping of spontaneous masculinization in XX doubled haploid rainbow trout lines," in Proceedings of the 10th World Congress on Genetics Applied to Livestock Production (WCGALP)), Vancouver, BC.

Harvey, A. C., Skilbrei, O. T., Besnier, F., Solberg, M. F., Sorvik, A. G. E., and Glover, K. A. (2018). Implications for introgression: has selection for fast growth altered the size threshold for precocious male maturation in domesticated Atlantic salmon? BMC Evol. Biol. 18:188. doi: 10.1186/s12862-018-1294-y

Harvey, A. C., Solberg, M. F., Troianou, E., Carvalho, G. R., Taylor, M. I., Creer, S., et al. (2016). Plasticity in growth of farmed and wild Atlantic salmon: is the increased growth rate of farmed salmon caused by evolutionary adaptations to the commercial diet? BMC Evol. Biol. 16:264. doi: 10.1186/s12862-016-0841-7 
Hattori, R. S., Somoza, G. M., Fernandino, J. I., Colautti, D. C., Miyoshi, K., Gong, Z., et al. (2019). The duplicated Y-specific amhy gene is conserved and linked to maleness in silversides of the genus Odontesthes. Genes 10:679. doi: 10.3390/genes 10090679

Heule, C., Salzburger, W., and Bohne, A. (2014). Genetics of sexual development: an evolutionary playground for fish. Genetics 196, 579-591. doi: 10.1534/ genetics.114.161158

Kijas, J., McWilliam, S., Sanchez, M. N., Kube, P., King, H., Evans, B., et al. (2018). Evolution of sex determination loci in Atlantic salmon. Sci. Rep. 8:5664. doi: 10.1038/s41598-018-23984-1

King, R. A., and Stevens, J. R. (2020). An improved genetic sex test for Atlantic salmon (Salmo salar L.). Conserv. Genet. Resour. 12, 191-193. doi: 10.1007/ s12686-019-01094-y

Kjaerner-Semb, E., Ayllon, F., Furmanek, T., Wennevik, V., Dahle, G., Niemela, E., et al. (2016). Atlantic salmon populations reveal adaptive divergence of immune related genes - a duplicated genome under selection. BMC Genomics 17:610. doi: 10.1186/s12864-016-2867-z

Kusche, H., Côté, G., Hernandez, C., Normandeau, E., Boivin-Delisle, D., and Bernatchez, L. (2017). Characterization of natural variation in North American Atlantic salmon populations (Salmonidae: Salmo salar) at a locus with a major effect on sea age. Ecol. Evol. 7, 5797-5807. doi: 10.1002/ece3. 3132

Larson, W. A., McKinney, G. J., Seeb, J. E., and Seeb, L. W. (2016). Identification and characterization of sex-associated loci in sockeye salmon using genotypingby-sequencing and comparison with a sex-determining assay based on the $s d Y$ gene. J. Hered. 107, 559-566. doi: 10.1093/jhered/esw043

Lien, S., Gidskehaug, L., Moen, T., Hayes, B. J., Berg, P. R., Davidson, W. S., et al. (2011). A dense SNP-based linkage map for Atlantic salmon (Salmo salar) reveals extended chromosome homeologies and striking differences in sex-specific recombination patterns. BMC Genomics 12:615. doi: 10.1186/14712164-12-615

Lien, S., Koop, B. F., Sandve, S. R., Miller, J. R., Kent, M. P., Nome, T., et al. (2016). The Atlantic salmon genome provides insights into rediploidization. Nature 533, 200-205. doi: 10.1038/nature17164

Livak, K. J., and Schmittgen, T. D. (2001). Analysis of relative gene expression data using real-time quantitative PCR and the 2(T)(-Delta Delta C) method. Methods 25, 402-408. doi: 10.1006/meth.2001.1262

Lubieniecki, K. P., Lin, S., Cabana, E. I., Li, J. Y., Lai, Y. Y. Y., and Davidson, W. S. (2015). Genomic instability of the sex-determining locus in Atlantic salmon (Salmo salar). G3 (Bethesda) 5, 2513-2522. doi: 10.1534/g3.115. 020115

Macqueen, D. J., and Johnston, I. A. (2014). A well-constrained estimate for the timing of the salmonid whole genome duplication reveals major decoupling from species diversification. Proc. R. Soc. B-Biol. Sci. 281:20132881. doi: 10. 1098/rspb.2013.2881

Metcalf, V. J., and Gemmell, N. J. (2006). Sexual genotype markers absent from small numbers of male New Zealand Oncorhynchus tshawytscha. J. Fish Biol. 68, 136-143. doi: 10.1111/j.0022-1112.2006. 00903.x

Nagler, J. J., Bouma, J., Thorgaard, G. H., and Dauble, D. D. (2001). High incidence of a male-specific genetic marker in phenotypic female chinook salmon from the Columbia River. Environ. Health Perspect. 109, 67-69. doi: 10.1289/ehp. 0110967

Pendas, A. M., Moran, P., Martinez, J. L., and Garciavazquez, E. (1995). Applications of 5S-rDNA in Atlantic salmon, brown trout, and in Atlantic salmon x brown trout hybrid identification. Mol. Ecol. 4, 275-276. doi: 10.1111/ j.1365-294X.1995.tb00220.x
Pennell, M. W., Mank, J. E., and Peichel, C. L. (2018). Transitions in sex determination and sex chromosomes across vertebrate species. Mol. Ecol. 27, 3950-3963. doi: 10.1111/mec. 14540

Perry, W. B., Solberg, M. F., Besnier, F., Dyrhovden, L., Matre, I. H., Fjelldal, P. G., et al. (2019). Evolutionary drivers of kype size in Atlantic salmon (Salmo salar): domestication, age and genetics. R. Soc. Open Sci. 6:190021. doi: 10.1098/rsos. 190021

Phillips, R. B. (2013). Evolution of the sex chromosomes in salmonid fishes. Cytogenet. Genome Res. 141, 177-185. doi: 10.1159/000355149

Podlesnykh, A. V., Brykov, V. A., and Kukhlevsky, A. D. (2017). Unstable linkage of molecular markers with sex determination gene in Pacific salmon (Oncorhynchus spp.). J. Hered. 108, 328-333. doi: 10.1093/jhered/esx001

Pong-Wong, R., George, A. W., Woolliams, J. A., and Haley, C. S. (2001). A simple and rapid method for calculating identity-by-descent matrices using multiple markers. Genet. Sel. Evol. 33, 453-471. doi: 10.1186/1297-9686-33-5-453

Qian, W. F., and Zhang, J. Z. (2014). Genomic evidence for adaptation by gene duplication. Genome Res. 24, 1356-1362. doi: 10.1101/gr.172098.114

R Development Core Team (2019). R: A Language and Environment for Statistical Computing. Vienna: R Foundation for Statistical Computing.

Ross, J. A., Urton, J. R., Boland, J., Shapiro, M. D., and Peichel, C. L. (2009). Turnover of sex chromosomes in the stickleback fishes (Gasterosteidae). PLoS Genet. 5:e1000391. doi: 10.1371/journal.pgen.1000391

Solberg, M. F., Fjelldal, P. G., Nilsen, F., and Glover, K. A. (2014). Hatching time and alevin growth prior to the onset of exogenous feeding in farmed, wild and hybrid Norwegian Atlantic salmon. PLoS One 9:e113697. doi: 10.1371/journal. pone.0113697

Solberg, M. F., Glover, K. A., Nilsen, F., and Skaala, Ø (2013). Does domestication cause changes in growth reaction norms? A study of farmed, wild and hybrid Atlantic salmon families exposed to environmental stress. PLoS One 8:e54469. doi: 10.1371/journal.pone.0054469

Taggart, J. B. (2007). FAP: an exclusion-based parental assignment program with enhanced predictive functions. Mol. Ecol. Notes 7, 412-415. doi: 10.1111/j.14718286.2006.01616.x

Williamson, K. S., and May, B. (2002). Incidence of phenotypic female chinook salmon positive for the male Y-chromosome-specific marker OtY1 in the Central Valley, California. J. Aquat. Anim. Health 14, 176-183. doi: 10.1577/ 1548-86672002014<0176:IOPFCS<2.0.CO;2

Yano, A., Guyomard, R., Nicol, B., Jouanno, E., Quillet, E., Klopp, C., et al. (2012). An immune-related gene evolved into the master sex-determining gene in rainbow trout, Oncorhynchus mykiss. Curr. Biol. 22, 1423-1428. doi: 10.1016/ j.cub.2012.05.045

Yano, A., Nicol, B., Jouanno, E., Quillet, E., Fostier, A., Guyomard, R., et al. (2013). The sexually dimorphic on the Y-chromosome gene $(s d Y)$ is a conserved malespecific Y-chromosome sequence in many salmonids. Evol. Appl. 6, 486-496. doi: $10.1111 /$ eva. 12032

Conflict of Interest: The authors declare that the research was conducted in the absence of any commercial or financial relationships that could be construed as a potential conflict of interest.

Copyright (c) 2020 Ayllon, Solberg, Besnier, Fjelldal, Hansen, Wargelius, Edvardsen and Glover. This is an open-access article distributed under the terms of the Creative Commons Attribution License (CC BY). The use, distribution or reproduction in other forums is permitted, provided the original author(s) and the copyright owner(s) are credited and that the original publication in this journal is cited, in accordance with accepted academic practice. No use, distribution or reproduction is permitted which does not comply with these terms. 